\title{
Rapid function approximation by modified Fourier series
}

Daan Huybrechs and Sheehan Olver

\begin{abstract}
We review a set of algorithms and techniques to approximate smooth functions on a domain $\Omega \subset \mathbb{R}^{d}$ by an expansion in eigenfunctions of the Laplacian. We refer to such expansions as modified Fourier series. These series converge pointwise everywhere in the domain of approximation, including on the boundary, at an algebraic rate that is essentially arbitrary. The computational complexity of the transformation is only linear in the number of terms of the expansion. Moreover, additional terms can be computed adaptively and efficiently.
\end{abstract}

\subsection{Introduction}

The subject of this review paper is the approximation of smooth functions. The method of approximation is quite simple: a smooth function $f$ on a domain $\Omega$ is expanded into eigenfunctions $u_{n}$ of the Laplace operator, subject to Neumann boundary conditions:

$$
\begin{aligned}
-\Delta u_{n}(x) & =\lambda_{n} u_{n}(x), & & x \in \Omega, \\
\frac{\partial u_{n}}{\partial \nu}(x) & =0, & & x \in \partial \Omega .
\end{aligned}
$$

On the face of it, this may not appear to be a recent research topic. It has long been known that such eigenfunctions are orthogonal and dense in $L_{2}[\Omega]$, making them ideally suitable for series expansions of quite arbitrary functions on domains with great generality. The Laplace operator has probably received the widest study among all partial differential operators. Yet, for the purpose of numerical approximation, it is usually neglected in favour of alternative approaches. Two well-known 
and successful examples for univariate functions are the approximation by Chebyshev polynomials, for smooth functions on an interval, and the FFT algorithm, for smooth functions that are in addition periodic. The recent research in modified Fourier series brings two properties that are indispensable for any approximation method: fast algorithms and rapid convergence, thereby adding competitiveness to generality.

One of the main results is an $\mathcal{O}(m)$ algorithm for the computation of the first $m$ coefficients in the series. This result holds for periodic and non-periodic functions alike. Needless to say, this computational complexity compares favourably to the alternatives mentioned above. The result has been made possible primarily through the advent of efficient computational schemes for highly oscillatory integrals. Such schemes are the subject of a separate review paper in this same volume, to which the interested reader is referred [9]. The connection to modified Fourier series becomes obvious once we observe that Laplace-Neumann eigenfunctions become increasingly oscillatory. Hence, the coefficients of the series are given by increasingly oscillatory integrals. The most important development in the evaluation of highly oscillatory integrals is, arguably, the Filon-type method [10]. We will show how Filon-type methods can be extended and adapted to the setting of modified Fourier series. The $\mathcal{O}(m)$ algorithm then follows immediately.

The second property is rapid convergence. In unaltered form, the algorithm briefly described above yields $\mathcal{O}\left(\mathrm{m}^{-2}\right)$ convergence, pointwise in the interior of $\Omega$, and $\mathcal{O}\left(m^{-1}\right)$ convergence on the boundary $\partial \Omega$. Note that the FFT-algorithm on an interval $[a, b]$, though ideally suited for periodic functions, yields only $\mathcal{O}\left(\mathrm{m}^{-1}\right)$ pointwise convergence for non-periodic functions in the interior, and no convergence at all at the boundary points. The convergence of modified Fourier series can be improved in several ways. In this paper we discuss two approaches: faster initial convergence through the use of eigenfunctions of polyharmonic operators and accelerated convergence through the use of polynomial subtraction. A possible third approach is based on extrapolation [14].

The main results in the approximation by modified Fourier series have been established in a series of papers $[11,12,13,14,8]$. The current paper mostly follows the same pattern of developments. We start by reviewing one-dimensional approximation in $§ 1.2$. The generalization in the direction of polyharmonic operators is discussed in $\$ 1.3$. A generalization in a different direction, to multivariate approximation, is re- 
viewed in $\S 1.4$. Finally, we treat the acceleration of convergence in $\S 1.5$. We end with some concluding remarks in $\S 1.6$.

\subsection{Univariate approximation}

The simplest setting is that of a function $f$ defined on the interval $\Omega:=$ $[-1,1]$. This univariate setting is well suited to motivate the use of Laplace-Neumann expansions and to appreciate their basic properties.

\subsubsection{Laplace-Neumann expansions}

The standard Fourier series of $f$ on the interval $[-1,1]$ is given by

$$
\frac{1}{2} \hat{f}_{0}^{C}+\sum_{n=1}^{\infty} \hat{f}_{n}^{C} \cos \pi n x+\hat{f}_{n}^{D} \sin \pi n x,
$$

where

$$
\hat{f}_{n}^{C}=\int_{-1}^{1} f(x) \cos \pi n x \mathrm{~d} x, \quad \hat{f}_{n}^{D}=\int_{-1}^{1} f(x) \sin \pi n x \mathrm{~d} x .
$$

Let us assume that the function $f$ is non-periodic. In that case, the Fourier coefficients as defined above behave like $\hat{f}_{n}^{C}=\mathcal{O}\left(n^{-2}\right)$ and $\hat{f}_{n}^{D}=$ $\mathcal{O}\left(n^{-1}\right)$ for $n \gg 1$. We note that the sine coefficients are primarily responsible for the slow convergence rate of the Fourier series.

The expansion in eigenfunctions of the Laplace operator, as defined by (1.1), leads to a very similar series:

$$
\frac{1}{2} \hat{f}_{0}^{C}+\sum_{n=1}^{\infty} \hat{f}_{n}^{C} \cos \pi n x+\hat{f}_{n}^{S} \sin \pi\left(n-\frac{1}{2}\right) x,
$$

where

$$
\hat{f}_{n}^{S}=\int_{-1}^{1} f(x) \sin \pi\left(n-\frac{1}{2}\right) x \mathrm{~d} x .
$$

The only difference compared to classical Fourier is a shift by $\frac{1}{2}$ in the argument of the sine functions, hence the name modified Fourier series. This small change suffices to yield $\mathcal{O}\left(n^{-2}\right)$ behaviour of the corresponding coefficients in the series when $f$ is differentiable and its derivative has bounded variation. Moreover, both $\hat{f}_{0}^{C}$ and $\hat{f}_{0}^{S}$ have an even expansion if $f$ is sufficiently smooth. This follows from integration by parts and establishes a pattern for the upcoming generalizations. 


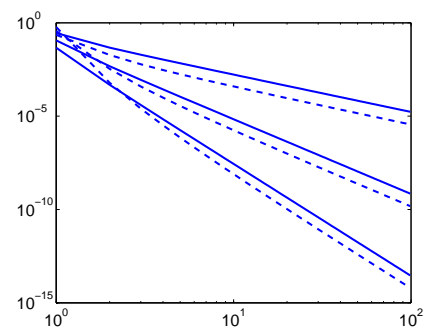

(a) Absolute size of the coefficients $\hat{f}_{n}^{C}$ (full lines) and $\hat{f}_{n}^{S}$ (dashed lines)

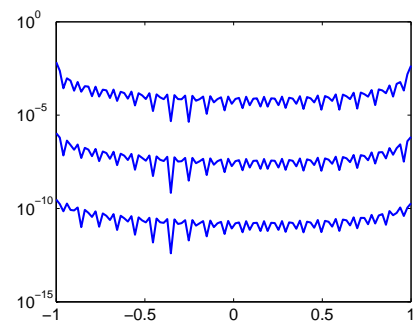

(b) Approximation error for $m=30$ terms on the interval $(-1,1)$

Fig. 1.1. Approximation of $f(x)=\cos (x+1) \sin (x+1)$ by a modified Fourier series. Polynomial subtraction was used with 0,1 and 2 steps (from top to bottom), leading to convergence rates of $\mathcal{O}\left(m^{-2}\right), \mathcal{O}\left(m^{-4}\right)$ and $\mathcal{O}\left(m^{-6}\right)$.

Theorem 1.1 If $f \in C^{\infty}[-1,1]$ then for $n \gg 1$ we have

$$
\begin{aligned}
& \hat{f}_{n}^{C} \sim(-1)^{n} \sum_{k=0}^{\infty} \frac{(-1)^{k}}{(n \pi)^{2 k+2}}\left[f^{(2 k+1)}(1)-f^{(2 k+1)}(-1)\right], \\
& \hat{f}_{n}^{S} \sim(-1)^{n-1} \sum_{k=0}^{\infty} \frac{(-1)^{k}}{\left[\left(n-\frac{1}{2}\right) \pi\right]^{2 k+2}}\left[f^{(2 k+1)}(1)+f^{(2 k+1)}(-1)\right] .
\end{aligned}
$$

Proof Integrating expression (1.3) by parts once yields

$$
\hat{f}_{n}^{S}=-\left.\frac{f(x) \cos \pi\left(n-\frac{1}{2}\right) x}{\left(n-\frac{1}{2}\right) \pi}\right|_{-1} ^{1}+\frac{1}{\left(n-\frac{1}{2}\right) \pi} \int_{-1}^{1} f^{\prime}(x) \cos \pi\left(n-\frac{1}{2}\right) x \mathrm{~d} x .
$$

The first term vanishes due to the homogeneous Neumann boundary conditions. Integrating by parts once more leads to

$\hat{f}_{n}^{S}=\left.\frac{f^{\prime}(x) \sin \pi\left(n-\frac{1}{2}\right) x}{\left(n-\frac{1}{2}\right)^{2} \pi^{2}}\right|_{-1} ^{1}-\frac{1}{\left(n-\frac{1}{2}\right)^{2} \pi^{2}} \int_{-1}^{1} f^{(2)}(x) \sin \pi\left(n-\frac{1}{2}\right) x \mathrm{~d} x$.

Repeated invocation of integration by parts on the remainder integral yields the result. The proof for the coefficients $\hat{f}_{n}^{C}$ is analogous.

It is thus established that the coefficients of the modified Fourier series (1.2) decay faster than those of a classic Fourier series. We emphasize the fact that this property essentially follows from the homogeneous Neumann boundary conditions satisfied by the basis functions.

The asymptotic expansions in Theorem 1.1 also indicate that the coefficients asymptotically depend only on odd derivatives of $f$, evaluated at the two boundary points of the interval $[-1,1]$. This observation will be used later on for efficiently accelerating the decay even further. 
The size of the coefficients and the approximation error are illustrated in Figure 1.1 (top curve). The figure also shows improved decay rates through polynomial subtraction. This will be discussed later in $§ 1.5 .1$.

\subsubsection{Convergence of the modified Fourier series}

The Laplace-Neumann eigenfunctions in the univariate case are given by the set of basis functions

$$
\{\cos \pi n x: n \geq 0\} \cup\left\{\sin \pi\left(n-\frac{1}{2}\right) x: n \geq 1\right\} .
$$

These functions form an orthonormal set in $L_{2}[-1,1]$. It follows that convergence of the modified Fourier series

$$
f_{m}(x):=\sum_{n=0}^{m} \hat{f}_{n}^{C} \cos \pi n x+\sum_{n=1}^{m} \hat{f}_{n}^{S} \sin \pi\left(n-\frac{1}{2}\right) x
$$

to $f$ is guaranteed in the $L_{2}$-norm. However, a more interesting notion in function approximation is pointwise convergence. In this section we review the known results on pointwise convergence of the series (1.5) to the value $f(x)$.

Theorem 1.2 ([11]) Suppose that $f$ is Riemann integrable in $[-1,1]$ and that

$$
\hat{f}_{n}^{C}, \hat{f}_{n}^{S}=\mathcal{O}\left(n^{-1}\right), \quad n \rightarrow \infty .
$$

If $f$ is Lipschitz at $x \in(-1,1)$ then

$$
f_{m}(x) \rightarrow f(x)
$$

The result is proved by amending the classical theorems of Féjer and of de la Vallée Poussin. The rate of convergence of the series was later established in [14]. We quote the following theorem.

Theorem 1.3 ([14]) Suppose that $f \in \mathcal{C}^{2}[-1,1]$ and $f^{\prime \prime}$ has bounded variation. If $-1<x<1$ then

$$
f(x)-f_{m}(x)=\mathcal{O}\left(m^{-2}\right) .
$$

Otherwise,

$$
f( \pm 1)-f_{m}( \pm 1)=\mathcal{O}\left(m^{-1}\right) .
$$




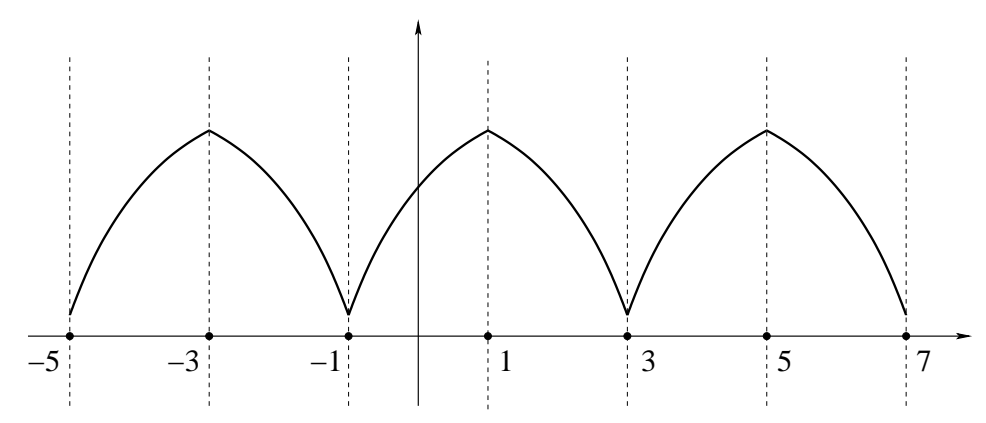

Fig. 1.2. The function $f(x)$ on $[-1,1]$ is evenly reflected around the points \pm 1 and extended periodically.

The series converges everywhere, though at a slower rate in the endpoints $x= \pm 1$. The result was obtained by expanding the difference $f(x)-f_{m}(x)$ asymptotically in terms of the special Lerch function. An asymptotic expansion for the approximation error was thus constructed that is uniform for $x \in[-1,1]$, i.e., including the endpoints. This opens the possibility of increasing the convergence rate even further, by explicitly adding the first term of the error expansion. We do not delve into this topic further in this paper, but refer the interested reader to [14].

\subsubsection{A geometric interpretation}

Theorem 1.1 showed that the coefficients $\hat{f}_{n}^{C}$ and $\hat{f}_{n}^{S}$ asymptotically depend only on odd derivatives of $f$ at the boundary points \pm 1 . This may seem odd at first sight. In this section, we attempt to give a meaning to this result with a geometric argument. In the process, we obtain a close relation to classical Fourier series in this univariate case.

Consider the function $g(x)$, defined on the interval $[-1,3]$ and constructed from $f(x)$ by reflecting evenly around the point $x=1$,

$$
g(x)= \begin{cases}f(x), & \text { if } x \in[-1,1] \\ f(2-x), & \text { if } x \in(1,3]\end{cases}
$$

Next, we extend the function $g$ periodically as illustrated in Figure 1.2. Note that reflecting evenly around the point $x=-1$ and then periodically extending would result in the same function. 
Now consider the classical Fourier series of $g$ on the interval $[-1,3]$,

$$
g_{m}(x)=\sum_{n=0}^{m} \tilde{g}_{n}^{C} \cos \left(\frac{2 \pi}{4} n(x+1)\right)+\sum_{n=1}^{m} \tilde{g}_{n}^{S} \sin \left(\frac{2 \pi}{4} n(x+1)\right),
$$

where

$$
\begin{array}{ll}
\tilde{g}_{n}^{C}:=\int_{-1}^{3} g(x) \cos \left(\frac{2 \pi}{4} n(x+1)\right) \mathrm{d} x, & n=0,1, \ldots, \\
\tilde{g}_{n}^{S}:=\int_{-1}^{3} g(x) \sin \left(\frac{2 \pi}{4} n(x+1)\right) \mathrm{d} x, & n=1,2, \ldots
\end{array}
$$

Since $g(x)$ is even on $[-1,3]$ around the center point $x=1$, we have $\tilde{g}_{n}^{S}=0$. One can also easily verify that

$$
\hat{f}_{n}^{C}=(-1)^{n} \frac{\tilde{g}_{2 n}^{C}}{2}, \quad \hat{f}_{n}^{S}=(-1)^{n} \frac{\tilde{g}_{2 n-1}^{C}}{2} .
$$

In other words, the modified Fourier series of $f$ is equivalent to the classical Fourier series of $g$. Figure 1.2 now explains the importance of odd derivatives at \pm 1 . If $f^{\prime}(1)=f^{\prime}(-1)=0$, then one sees that the differentiability of $g$ across the reflection points is increased. Thus, its Fourier series converges faster. The values of the even derivatives of $f$ at \pm 1 are irrelevant, as they do not limit the continuity of $g$.

We observe that the modified Fourier series of $f$ converges faster if $f$ can be extended as a smooth and even function across the boundary of the domain $\Omega$. We will see that this observation generalizes to different domains $\Omega$. The analogy to Fourier series, unfortunately, does not.

Due to this analogy with Fourier series, one may be tempted to compute the coefficients $\hat{f}_{n}^{C}$ and $\hat{f}_{n}^{S}$ using FFT. The alternative approaches discussed in the next section are far more accurate however, because the function $g$ in general will not be a smooth function.

\subsubsection{Computation of the coefficients}

The coefficients $\hat{f}_{n}^{C}$ and $\hat{f}_{n}^{S}$ are given by integrals that become increasingly oscillatory as $n \gg 1$. An account of recent research in efficient methods for the evaluation of oscillatory integrals is given in [9]. From the results in that review, it becomes apparent that the coefficients can be computed at a cost that is asymptotically independent of $n$. That is, each coefficient $\hat{f}_{n}^{C}$ and $\hat{f}_{n}^{S}$ can be computed with $O(1)$ evaluations of $f$ as $n \gg 1$. An immediate result is that the first $m$ coefficients can be computed in $O(m)$ operations.

An additional desire in the setting of modified Fourier series is to reuse 
function evaluations of $f$ for the computation of coefficients with varying values of $n$. With this goal in mind, we will revisit Filon-type quadrature, though noting that Levin-type methods are equally applicable [14].

The purpose of so-called exotic quadrature is again to maximize reuse of function evaluations, this time for the computation of coefficients with smaller $n$, in a further attempt to bridge the gap between oscillatory and non-oscillatory quadrature.

\subsubsection{Filon-type quadrature}

The simplest approximation method for $\hat{f}_{n}^{C}$ and $\hat{f}_{n}^{S}$ is, arguably, a truncation of the asymptotic expansions in Theorem 1.1. For example, we may define

$$
\hat{A}_{s, n}^{C}[f] \sim(-1)^{n} \sum_{k=0}^{s-1} \frac{(-1)^{k}}{(n \pi)^{2 k+2}}\left[f^{(2 k+1)}(1)-f^{(2 k+1)}(-1)\right],
$$

which corresponds to truncating the expansion for $\hat{f}_{n}^{C}$ after $s$ terms. This approximation carries an error of asymptotic order

$$
\hat{f}_{n}^{C}-\hat{A}_{s, n}^{C}[f] \sim \mathcal{O}\left(n^{-2 s-2}\right), \quad n \gg 1 .
$$

The error has the same size, asymptotically, as the first discarded term in the expansion. It decreases rapidly as $n$ becomes large.

This asymptotic approximation is not very useful however for small values of $n$. The idea of Filon-type quadrature is to maintain high asymptotic order, in the sense of (1.6), while also maintaining the classical notion of polynomial exactness. That is, Filon-type quadrature rules are exact when $f$ is a polynomial of a certain degree. For general oscillatory integrals, this leads to quadrature rules involving derivatives. Let

$$
-1=c_{1}<c_{2}<\ldots<c_{\nu}=1
$$

be a set of $\nu$ quadrature points with associated multiplicities $m_{k} \in \mathbb{N}$. We construct a polynomial $p$ of degree $\sum_{k=1}^{\nu} m_{k}-1$ such that

$$
p^{(i)}\left(c_{k}\right)=f^{(i)}\left(c_{k}\right), \quad i=0,1, \ldots, m_{k}-1, \quad k=1,2, \ldots, \nu .
$$

Then a Filon-type method may be defined by

$$
\hat{f}_{n}^{C} \approx \hat{Q}_{n}^{C}[f]:=\hat{p}_{n}^{C}=\int_{-1}^{1} p(x) \cos \pi n x \mathrm{~d} x .
$$

This can be expressed in closed form since the moments are known, 
which are computable either via integration by parts or the formula (for $k>0)$ :

$$
\begin{gathered}
\int_{-1}^{1} x^{k} \cos \pi n x \mathrm{~d} x= \\
\Re\left\{(-i n \pi)^{-k-1}[\Gamma(1+k, i n \pi)-\Gamma(1+k,-i n \pi)]\right\},
\end{gathered}
$$

where $\Gamma$ is the incomplete Gamma function.

In the context of modified Fourier series, this general setting changes in the following way. The key to obtain high asymptotic order is to interpolate the odd derivatives of $f$ at the endpoints. In other words, it is sufficient to interpolate precisely the data on which the early terms in the asymptotic expansion depend. This leads to a set of interpolation conditions of the form

$$
p^{(2 i+1)}\left(c_{k}\right)=f^{(2 i+1)}\left(c_{k}\right), \quad i=0,1, \ldots, m_{k}-1, \quad k=1,2, \ldots, \nu .
$$

Augmented by the condition $p(0)=f(0)$, this interpolation problem has a unique solution. The corresponding quadrature rules take the form

$$
\hat{Q}_{n}^{C}[f]:=\sum_{k=1}^{\nu} \sum_{j=0}^{m_{k}-1} \theta_{k, j}^{C}(n) f^{(2 j+1)}\left(c_{k}\right) .
$$

This rule has asymptotic error $\mathcal{O}\left(n^{-2 s-2}\right)$ if $m_{1}, m_{\nu} \geq s$. Similar rules can of course be constructed for the sine coefficients $\hat{f}_{n}^{S}$.

The weights $\theta_{k, j}^{C}(n)$ typically depend on $n$ in an explicit manner. Substantial insights in the design of Filon-type quadrature methods are developed in $[11,12,13]$, and we refer the reader to those papers for explicit examples of suitable quadrature rules.

\subsubsection{Exotic quadrature}

For small values of $n$, the integrals $\hat{f}_{n}^{C}$ and $\hat{f}_{n}^{S}$ to compute are nonoscillatory in nature. One can resort to any of the known quadrature schemes for smooth and non-oscillatory functions, such as composite Gaussian quadrature [6]. Note that for achieving an $O(m)$ algorithm for the computation of the first $m$ coefficients, it is actually irrelevant how the first (finitely many) elements are computed. Nevertheless, one naturally seeks for optimal methods that reduce computation time.

Given that the computation of coefficients for large $n$ requires derivatives at the endpoints, one can reuse this information in the computation of coefficients for small $n$. This leads to quadrature rules involving 
derivatives that, for lack of an established name, were dubbed exotic quadrature in [12]. They have the general form

$$
\int_{-1}^{1} g(x) \mathrm{d} x \approx \hat{P}[f]:=\sum_{k=1}^{\nu} \sum_{j \in N_{m_{k}}} \delta_{k, j} g^{(j)}\left(c_{k}\right),
$$

and they are typically applied to the function $g(x)=f(x) u_{n}(x)$, where $u_{n}(x)$ is one of the Laplace-Neumann eigenfunctions. We have introduced the sets $N_{m}$ to illustrate that the information about derivatives in exotic quadrature may be lacunary. For example, in the case of univariate modified Fourier series, we may define

$$
N_{m}:=\{2 j+1\}_{j=0}^{m-1} .
$$

The main message embodied in the theory of Filon-type quadrature and exotic quadrature is that the onset of asymptotic behaviour for increasing $n$ is quite rapid. It appears that asymptotic accuracy kicks in for very moderate values of $n$. It is only reasonable to exploit this.

\subsection{Polyharmonic approximation}

The importance of imposing homogeneous Neumann boundary conditions in the general setting (1.1) became visible in Theorem 1.1. The boundary conditions rendered the first term in the asymptotic expansion of the coefficients zero, thus generating faster decay. This observation leads in a natural way to the first generalization of the theory, where faster convergence is achieved by imposing higher-order Neumann boundary conditions. In particular, in this section we are interested in eigenfunctions of the polyharmonic operator

$$
u^{(2 q)}+(-1)^{q+1} \alpha^{2 q} u=0, \quad-1 \leq x \leq 1,
$$

subject to the Neumann boundary conditions

$$
u^{(i)}(-1)=u^{(i)}(1)=0, \quad i=q, q+1, \ldots, 2 q-1 .
$$

Here $q$ is a fixed parameter determining the order of the polyharmonic operator.

Denote the $n$th eigenvalue as

$$
\kappa_{n}=(-1)^{q} \alpha^{2 q},
$$


with the corresponding $n$th eigenfunction denoted as $u_{n}$. Like the modified Fourier series, the basis of eigenfunctions $\left\{u_{1}, u_{2}, \ldots\right\}$ form an orthogonal series with respect to the $L_{2}$ inner product. Furthermore, they are dense in $L_{2}[-1,1]$ [12]. Thus we can successfully utilize them for function approximation, giving us the expansion

$$
f(x) \sim \sum_{n=1}^{\infty} \hat{f}_{n} u_{n}, \quad \text { for } \quad \hat{f}_{n}=\int_{-1}^{1} f(x) u_{n}(x) \mathrm{d} x .
$$

By repeatedly utilizing integration by parts and assuming that $f$ is $(q+$ 1)-times differentiable, we immediately find that

$$
\begin{aligned}
\hat{f}_{n}= & \int_{-1}^{1} f(x) u_{n}(x) \mathrm{d} x=\frac{(-1)^{q}}{\alpha_{n}^{2 q}} \int_{-1}^{1} f(x) u_{n}^{(2 q)}(x) \mathrm{d} x \\
= & \frac{1}{\alpha_{n}^{2 q}} \int_{-1}^{1} f^{(q)}(x) u_{n}^{(q)}(x) \mathrm{d} x \\
= & \frac{1}{\alpha_{n}^{2 q}}\left[f^{(q)}(1) u_{n}^{(q-1)}(1)-f^{(q)}(-1) u_{n}^{(q-1)}(-1)\right] \\
& \quad-\frac{1}{\alpha_{n}^{2 q}} \int_{-1}^{1} f^{(q+1)}(x) u_{n}^{(q-1)}(x) \mathrm{d} x .
\end{aligned}
$$

From [12], we know that $u_{n}^{(i)}(x)=\mathcal{O}\left(\alpha_{n}^{i}\right)$. Furthermore, we also know that $\alpha_{n} \sim \mathcal{O}(n)$ [15]. It follows immediately that

$$
\hat{f}_{n}=\mathcal{O}\left(n^{-q-1}\right) .
$$

Thus we can obtain any algebraic convergence rate by choosing $q$ large enough.

\subsubsection{The basis of eigenfunctions}

In this section we will demonstrate how $\alpha_{n}$ and the eigenfunctions $u_{n}$ can be found. For simplicity we focus on the case where $q=2$, referring the interested reader to [12] for the derivation for other values of $q$. The first two eigenfunctions are trivial (which we will not include in the enumeration $\left.u_{1}, u_{2}, \ldots\right)$ : 1 and $x$. It follows immediately from (1.8) that the other eigenfunctions can be expressed as a sum of exponentials. In particular, for $q=2$ we obtain

$$
u(x)=c_{1} \cos \alpha x+c_{2} \sin \alpha x+c_{3} \cosh \alpha x+c_{4} \sinh \alpha x .
$$

Our goal, then, is to find which values of $c_{k}$ and $\alpha$ satisfy the boundary 


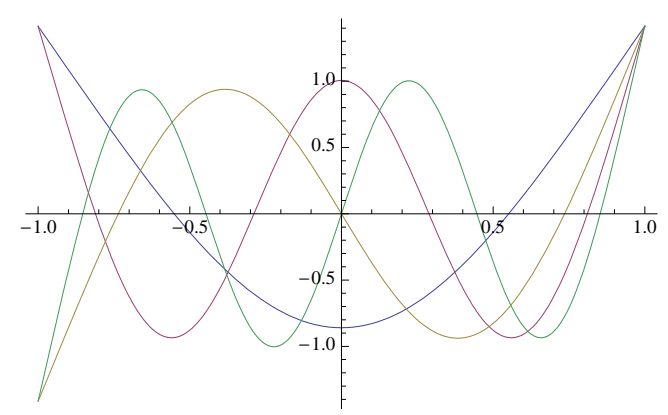

Fig. 1.3. The functions $u_{1}, \ldots, u_{4}$ on $[-1,1]$ for $q=2$.

conditions. Thus we want to ensure that $0=u^{\prime \prime}( \pm 1)=u^{\prime \prime \prime}( \pm 1)$. Sparing the algebraic details, this is achieved when

$$
\begin{aligned}
& u(x)=\frac{\sqrt{2}}{2}\left(\frac{\cos \alpha x}{\cos \alpha}+\frac{\cosh \alpha x}{\cosh \alpha}\right) \quad \text { if } \quad \tan \alpha+\tanh \alpha=0, \\
& u(x)=\frac{\sqrt{2}}{2}\left(\frac{\sin \alpha x}{\sin \alpha}+\frac{\sinh \alpha x}{\sinh \alpha}\right) \quad \text { if } \quad \tan \alpha-\tanh \alpha=0 .
\end{aligned}
$$

We are thus left with the problem of computing the roots of two transcendental equations. Trigonometric manipulations and calculus inform us that $\tan \alpha+\tanh \alpha$ has a root in each exponentially small window $\left[\left(n-\frac{1}{4}\right) \pi,\left(n-\frac{1}{4}\right) \pi+e^{-2\left(n-\frac{1}{4}\right) \pi}\right]$ for $n=1,2, \ldots$ Similarly, $\tan \alpha-\tanh \alpha$ has a root in $\left[\left(n+\frac{1}{4}\right) \pi-e^{-2\left(n+\frac{1}{4}\right) \pi},\left(n+\frac{1}{4}\right) \pi\right]$ for $n=1,2, \ldots$. These zeros interlace, and we can thus enumerate the zeros of each transcendental equation as $\alpha_{n}$, where a sufficiently small neighbourhood is known where the zero exists as to make its computation straightforward. Thus we can successfully compute the eigenfunction $u_{n}$ associated to $\alpha_{n}$, and in Figure 1.3 we graph the first four such eigenfunctions.

\subsection{Multivariate approximation}

The generalization of univariate modified Fourier expansions to a higherdimensional domain $\Omega \cup \mathbb{R}^{d}$ is, at least in principle, straightforward: the expansion is simply defined in terms of eigenfunctions of the Laplace operator on $\Omega$, subject to Neumann boundary conditions. We will show below that this indeed leads to rapid convergence in very general circumstances. Unfortunately, the set of domains for which the eigenfunctions are known with suitably explicit expressions is small. In practice, 
multivariate modified Fourier expansions are therefore limited to a few domains. In two dimensions, the list includes ellipses, rectangles, annuli and a number of different triangles.

\subsubsection{Laplace-Neumann eigenfunctions}

We are looking for eigenfunctions $u$ of the Laplace-Neumann problem in a simply-connected and bounded domain $\Omega \subset \mathbb{R}^{d}$,

$$
-\Delta u=\lambda u, \quad \mathbf{x} \in \Omega, \quad \frac{\partial u}{\partial \nu}=0, \quad \mathbf{x} \in \partial \Omega
$$

We can in general denote the countable set of eigenvalues and eigenfunctions by $\lambda_{n}$ and $u_{n}, n \geq 0$, with $\lambda_{k} \leq \lambda_{n}$ if $k<n$. It is always true that $\lambda_{0}=0$ and $u_{0} \equiv 1$, and all subsequent eigenvalues are strictly positive. With this ordering, the Weyl theorem holds:

$$
\lambda_{n} \sim \operatorname{meas}(\Omega) n^{\frac{2}{d}}, \quad n \gg 1,
$$

where meas $(\Omega)$ denotes the measure of $\Omega$ [5]. A function $f$ can be expanded in the series

$$
f \sim \sum_{n=0}^{\infty} \frac{\hat{f}_{n}}{\hat{u}_{n}} u_{n}
$$

where $\hat{u}_{n}=\left\langle u_{n}, u_{n}\right\rangle$ and

$$
\hat{f}_{n}=\left\langle f, u_{n}\right\rangle=\int_{\Omega} f(\mathbf{x}) u_{n}(\mathbf{x}) \mathrm{d} V .
$$

In practice, other notation is often more convenient, as explicitly known eigenfunctions in more than one dimension typically depend on more than one parameter. This of course depends on the case at hand. We will review the case of the $d$-dimensional cube in $\S 1.4 .3$. First however, we continue the general setting to discuss convergence.

\subsubsection{Convergence of the approximation}

Convergence of the series (1.11) implies decay of the coefficients. In one dimension, the rate of decay of the coefficients was established in Theorem 1.1 via integration by parts. The multivariate counterpart of integration by part is the Stokes theorem. This, in connection with the Weyl theorem, will again establish rapid decay of Laplace-Neumann coefficients in the general setting treated so far. 
Given $f \in C^{\infty}[\Omega]$, we replace $u_{n}$ with $\lambda^{-1} \Delta u_{n}$ in (1.12). Applying Stokes theorem twice and substituting homogeneous Neumann boundary conditions [13], this leads to

$$
\left\langle f, u_{n}\right\rangle=\frac{1}{\lambda_{n}} \int_{\partial \Omega} \frac{\partial f(\mathbf{x})}{\partial \nu} u_{n}(\mathbf{x}) \mathrm{d} S-\frac{1}{\lambda_{n}}\left\langle\Delta f, u_{n}\right\rangle .
$$

Iterating the approach, we obtain the expansion

$$
\left\langle f, u_{n}\right\rangle \sim-\sum_{m=0}^{\infty} \frac{1}{\left(-\lambda_{n}\right)^{m+1}} \int_{\partial \Omega} \frac{\partial \Delta^{m} f(\mathbf{x})}{\partial \nu} u_{n}(\mathbf{x}) \mathrm{d} S, \quad \lambda_{n} \gg 1 .
$$

We make the following remarks:

(i) (1.13) converges only in an asymptotic sense, that is for $\lambda_{n} \gg 1$ (or, equivalently, $n \gg 1$ ).

(ii) the size of the coefficient is given, asymptotically, by the first term in the expansion. Assuming orthonormal $u_{n}$, the integral remains bounded as $n \gg 1$. It follows that $\hat{f}_{n}=\mathcal{O}\left(\lambda_{n}^{-1}\right)=\mathcal{O}\left(n^{-\frac{2}{a}}\right)$ from the Weyl theorem.

(iii) the coefficients decay faster if $f$ can be extended as a smooth and even function across $\partial \Omega$ (recall $\S 1.2 .3$ ). This follows because, for such functions, the normal derivatives $\frac{\partial^{2 j+1} f}{\nu^{2 j+1}}$ of odd order vanish on $\partial \Omega, j=0,1, \ldots$. Consequently, one can show that the normal derivatives $\frac{\partial \Delta^{m} f}{\partial \nu}$ also vanish, $m=0,1, \ldots$

It appears that expansion (1.13) is very informative. Yet, at the same time it may also be misleading. Most coefficients are in fact much smaller than the $\frac{1}{\lambda_{n}}$ estimate obtained above. In particular, for most values of $n$, the boundary integrals in (1.13) are themselves oscillatory integrals. This means they are much smaller than $\mathcal{O}(1)$ as was assumed in (ii). They can typically be expanded further asymptotically, using either Stokes' theorem or partial integration on the piecewise smooth parts of $\partial \Omega$. The results depend on the particular shape of $\Omega$.

Such further expansion is possible only if $u_{n}$ is oscillatory along the boundary $\partial \Omega$ of the domain. This is not always the case, even if $n \gg 1$. This phenomenon gives rise to the so-called hyperbolic cross [2], to which we will return later in $\S 1.5$. 


\subsection{3 d-dimensional cubes}

The case of the $d$-dimensional cube is the most obvious setting for a generalization of modified Fourier series to higher dimension. In this case, the Laplace-Neumann eigenfunctions are given by a tensor-product of the univariate eigenfunctions. We illustrate the points raised in $\S 1.4 .2$ above with the two-dimensional square $\Omega=[-1,1]^{2}$.

As indicated earlier, it is convenient to switch notation and have eigenfunctions depend on two parameters $m$ and $n$, rather than just $n$. These parameters correspond to frequency in $x$ and $y$ direction respectively. There are four kinds of eigenfunctions,

$$
\begin{aligned}
& u_{m, n}^{[0,0]}(x, y)=\cos (\pi m x) \cos (\pi n y), \\
& u_{m, n}^{[0,0]}(x, y)=\cos (\pi m x) \sin \left[\pi\left(n-\frac{1}{2}\right) y\right], \\
& u_{m, n,}^{[0,0]}(x, y)=\sin \left[\pi\left(m-\frac{1}{2}\right) x\right] \cos (\pi n y), \\
& u_{m, n}^{[0,0]}(x, y)=\sin \left[\pi\left(m-\frac{1}{2}\right) x\right] \sin \left[\pi\left(n-\frac{1}{2}\right) y\right] .
\end{aligned}
$$

The eigenvalues corresponding to $u_{m, n}^{[0,0]}$, for example, are $\lambda_{m, n}^{[0,0]}:=\pi^{2}\left(m^{2}+\right.$ $n^{2}$ ). This means that the coefficients decay as

$$
\left\langle f, u_{m, n}^{[0,0]}\right\rangle \sim \frac{1}{\lambda_{m, n}^{[0,0]}} \sim \mathcal{O}\left(\frac{1}{m^{2}+n^{2}}\right), \quad m^{2}+n^{2} \gg 1 .
$$

This estimate is valid as soon as either $m \gg 1$ or $n \gg 1$. However, if both $m$ and $n$ are large, we actually have

$$
\left\langle f, u_{m, n}^{[0,0]}\right\rangle \sim \mathcal{O}\left(\frac{1}{m^{2} n^{2}}\right), \quad m, n \gg 1 .
$$

This follows from a further integration by parts on the boundary integrals in (1.13). The same procedure shows that such coefficients asymptotically depend only on certain partial derivatives of $f$ at the vertices.

In the $(m, n)$ plane, we conclude that coefficients near the edge $m=$ 0 behave as $\mathcal{O}\left(n^{-2}\right)$ and coefficients near the edge $n=0$ behave as $\mathcal{O}\left(m^{-2}\right)$. Elsewhere the coefficients are much smaller and behave as $\mathcal{O}\left(m^{-2} n^{-2}\right)$. Such setting leads to the typical shape of a hyperbolic cross, that is illustrated further on in Figure 1.4. Note that the decay of the coefficients compares to $\mathcal{O}\left(m^{-1} n^{-1}\right)$ had we considered a classical tensor-product Fourier series instead. 


\subsubsection{Computation of the coefficients}

The computation of coefficients in the multivariate case is more involved than constructing a Cartesian product generalization of the univariate quadrature. This is true even in the case of a $d$-dimensional cube. We focus briefly on two of the issues surrounding multivariate quadrature (cubature) in the setting of modified Fourier series.

Cubature is based on polynomial interpolation, but not all sets of points are suitable for such interpolation [4]. The interpolation problem can be elegantly circumvented in the design of Filon-type methods by considering a Filon-type method as a correction to the asymptotic method. We formally write

$$
\hat{Q}_{s, n}^{F}[f]=\hat{A}_{s, n}[f]+\hat{E}_{s, n}[f],
$$

where $\hat{A}_{s, n}[f]$ is the asymptotic expansion up to an order defined by $s$. The correction term $\hat{E}_{s, n}$ is found as the best approximation to the next terms of the expansion, based on the available function evaluations of $f$. This can be achieved using finite differences. All known Filon-type methods that are exact for polynomials fit this formalism.

The second issues arises in so-called edge coefficients. These are coefficients given by integrals that are non-oscillatory in some variables, but oscillatory in the others. For example, in the case of the square as defined above, edge coefficients are those coefficients $\hat{f}_{m, n}^{[0,0]}$ where $m \gg 1$ and $n \approx 1$, or $m \approx 1$ and $n \gg 1$. The issue can be resolved by combining oscillatory and non-oscillatory quadrature, for example combining Filon-type quadrature in $x$ with exotic quadrature in $y$. We refer the reader to [13] for an in-depth discussion.

\subsection{Convergence acceleration}

Modified Fourier series converge quite rapidly, at least faster than classical Fourier series, while yielding convergence everywhere. Yet, the convergence rate can also be improved to essentially arbitrarily high algebraic rates. The first method reviewed in $\S 1.3$, by considering polyharmonic operators, yields faster initial convergence of $O\left(m^{-q-1}\right)$ but does not scale well to high order. An alternative is acceleration of the standard Laplace-Neumann case. In the section we focus on acceleration through the techniques of polynomial subtraction. 


\subsubsection{Polynomial subtraction}

Asymptotic expansions of the coefficients, such as those given in Theorem 1.1 and in expression (1.13), are very revealing. Let us consider first the univariate case. The idea of polynomial subtraction is simple: we subtract from $f$ a polynomial such that the first few terms of the expansion vanish. Thus, the coefficients decay faster and the convergence rate improves likewise.

We set

$$
g(x)=f(x)-p_{s}(x), \quad x \in[-1,1],
$$

where $p_{s}(x)$ is a polynomial that satisfies

$$
p_{s}^{(2 j+1)}( \pm 1)=f^{(2 j+1)}( \pm 1), \quad j=0, \ldots, s-1 .
$$

Lemma 1.1 Consider the modified Fourier series $g_{m}(x)$ for $g$. We have

$$
g(x)-g_{m}(x)=\mathcal{O}\left(m^{-2 s-2}\right), \quad m \gg 1 .
$$

Proof Follows immediately from Theorem 1.1.

We approximate $f(x)$ by $p_{s}(x)+g_{m}(x)$ with the same error. Note that $p_{s}(x)$ itself is not a good approximation to $f(x)$. The convergence rate is $\mathcal{O}\left(m^{-2 s-2}\right)$ for $x \in(-1,1)$ and $\mathcal{O}\left(m^{-2 s-1}\right)$ for $x= \pm 1$. The Gibbs phenomenon is still present, but clearly it is much less severe: convergence at the endpoints is only slightly slower than in the interior of the domain. The improved decay of the coefficients and accelerated convergence of the series was already illustrated in Figure 1.1 in $\S 1.2$. There, we constructed $p_{1}$ and $p_{2}$ for the function $f(x)=\cos (x+1) \sin (x+1)$.

Of course, one is not forced to use a polynomial basis for $p_{s}(x)$. In any case, all that is required for acceleration is a suitable estimate of the odd derivatives of $f(x)$ at the endpoints.

\subsubsection{Subtraction in a multivariate setting}

The above generalizes to multivariate integration in the following way.

Based on the asymptotic expansion (1.13), we again set

$$
g(\mathbf{x})=f(\mathbf{x})-p_{s}(\mathbf{x}),
$$




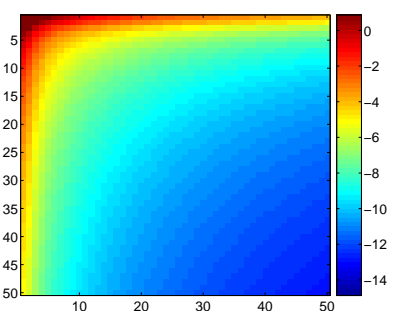

(a) No acceleration

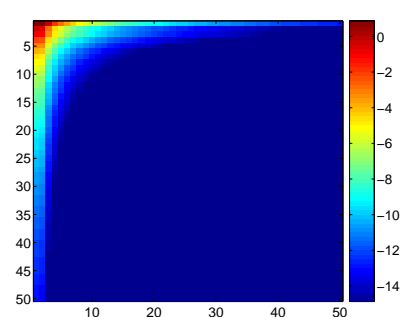

(b) Subtraction of 1 boundary term

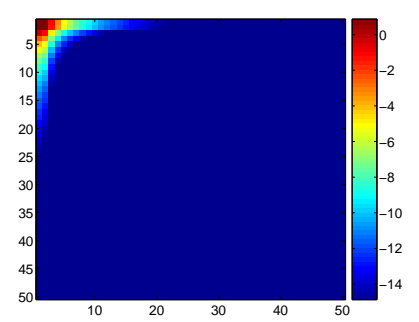

(c) Subtraction of 2 boundary terms

Fig. 1.4. The absolute value of coefficients $\hat{f}_{m, n}^{[0,0]}$ for $f(x)=e^{\pi(x-y)} \cos (x)$ with and without acceleration. The values are shown in base-10 logarithmic scale.

where now $p_{s}(\mathbf{x})$ is such that

$$
\frac{\partial \Delta^{j} p}{\partial \nu}(\mathbf{x})=\frac{\partial \Delta^{j} f}{\partial \nu}(\mathbf{x}), \quad \mathbf{x} \in \partial \Omega, \quad j=0, \ldots, s-1 .
$$

Lemma 1.2 Consider the modified Fourier series $g_{m}(\mathbf{x})$ for $g$. We have

$$
g(\mathbf{x})-g_{m}(\mathbf{x})=\mathcal{O}\left(\lambda_{m}^{-s-1}\right)
$$

Proof Follows immediately from (1.13).

Though the function $p_{s}(\mathbf{x})$ can in general not be a polynomial, the interpolation of a function or its derivatives along the boundary of a domain is a topic well studied in the field of Computer Aided Geometric Design (CAGD). A general construction for any $s$ in the case of $d$-dimensional cubes is given in [8].

Alternatively, it is easier to interpolate only partial derivatives at the vertices of the boundary $\partial \Omega$. This achieves accelerated decay of all coeffi- 
cients except for edge coefficients. The minimal set of partial derivatives to interpolate depends on the shape of $\Omega$.

We remark however that in boundary value problems, Neumann data is precisely the sort of information that may be available. The use of modified Fourier series in spectral methods for boundary value problems was recently explored in [1]. It was found that modified Fourier series are better behaved in this setting than Chebyshev expansions. In particular, the condition number of the discretization matrix behaves as $\mathcal{O}\left(\mathrm{m}^{2}\right)$ for modified Fourier series, but as $\mathcal{O}\left(m^{4}\right)$ for Chebyshev expansions.

Figure 1.4 illustrates the size of the modified Fourier coefficients for the case of the square $\Omega:=[-1,1]$ and the smooth function $f(x)=$ $e^{\pi(x-y)} \cos (x)$. The level curves roughly correspond to constant $m^{2} n^{2}$, due to the $\mathcal{O}\left(m^{-2} n^{-2}\right)$ behaviour of the coefficients. The hyperbolic cross derives from the hyperbolic shape of these curves. The hyperbolic cross is particularly interesting in higher dimension: one can show that the number of coefficients larger than a given threshold grows only logarithmically with dimension, rather than exponentially [8]. The acceleration procedure in this two-dimensional example is very effective indeed. For example, the number of elements larger than $10^{-5}$ in panel (a) is 2,113 , in panel (b) it is 184 and in panel (c) only 58. In the latter case, the coefficients behave as $O\left(m^{-6} n^{-6}\right)$, for $m, n \gg 1$.

\subsection{Concluding remarks}

At the beginning of this paper, we motivated the use of modified Fourier series by comparing to classical Fourier series and Chebyshev expansions. To summarize, we note the following advantages:

(i) The first $m$ coefficients can be computed in $\mathcal{O}(m)$ operations.

(ii) Additional coefficients can be computed adaptively, with an $\mathcal{O}(1)$ cost associated to each term.

(iii) The method of approximation generalizes in different directions: to eigenfunctions of polyharmonic operators and to general domains $\Omega$ including ellipses, cubes and triangles. (And, by extension, to any $\Omega$ that can be tessellated by these basic shapes. For example, any polygon can be divided in a set of triangles, and functions defined on a polygon can be approximated in each triangle separately.)

(iv) Modified Fourier series are more stable in spectral methods than 
Chebyshev expansions, exhibiting a typical $\mathcal{O}\left(\mathrm{m}^{2}\right)$ condition number of the discretization matrix rather than $\mathcal{O}\left(m^{4}\right)$.

Compared to FFT for non-periodic functions, modified Fourier series overcome the Gibbs phenomenon and yield pointwise convergence at the boundary. These facts are even more true when convergence acceleration is used. An alternative, and very effective, procedure for defeating the Gibbs phenomenon in one dimension was also reviewed in [7]. The described method achieves exponential accuracy in the reconstruction of a non-periodic function $f$ from its values in equidistant points, much like FFT for periodic functions, by expanding the partial Fourier sum into a set of Gegenbauer polynomials. The computations involved can become unstable however if $f$ has singularities close to the real axis [3]. Modified Fourier series on the other hand, leaving generality aside, are guaranteed to be stable for all domains $\Omega$ : all coefficients are easily bounded in terms of the maximum of $|f|$ on $\Omega$. Moreover, the techniques for convergence acceleration are quite flexible, and we anticipate that the degrees of freedom may be used in the future to produce numerically stable algorithms in a variety of applications.

\section{Bibliography}

[1] Adcock, B. (2007). Spectral methods and modified Fourier series, Technical Report 2007/NA08, University of Cambridge.

[2] Babenko, K. (1960). Approximation of periodic functions of many variables by trigonometric polynomials, Soviet Maths 1, 513-516.

[3] Boyd, J.P. (2005). Trouble with Gegenbauer reconstruction for defeating Gibbs' phenomenon: Runge phenomenon in the diagonal limit of Gegenbauer polynomial approximations, J. Comput. Phys. 204, 253-264.

[4] Cools, R. (1997). Constructing cubature formulae: The science behind the art, Acta Numer. 6, 1-54.

[5] Courant, R. and Hilbert, D. (1962). Methods of mathematical physics (Wiley Interscience, New York).

[6] Davis, P. J. and Rabinowitz, P. (1984). Methods of numerical integration, Computer Science and Applied Mathematics (Academic Press, New York).

[7] Gottlieb, D. and Shu, C.-W. (1997). On the Gibbs phenomenon and its resolution, SIAM Rev. 39, 644-668.

[8] Huybrechs, D., Iserles, A. and Nørsett, S. P. (2007). From high oscillation to rapid approximation IV: Accelerating convergence, Technical Report NA2007/07, University of Cambridge.

[9] Huybrechs, D. and Olver, S. (2008). Highly oscillatory quadrature, in Highly Oscillatory Problems: Computation, Theory and Applications, ed. B. Engquist et al (Cambridge University Press, Cambridge). 
[10] Iserles, A. and Nørsett, S. P. (2005). Efficient quadrature of highly oscillatory integrals using derivatives, Proc. R. Soc. Lond. A 461, 1383-1399.

[11] Iserles, A. and Nørsett, S. P. (2006). From high oscillation to rapid approximation I: Modified Fourier expansions, Technical Report 2006/NA05, University of Cambridge.

[12] Iserles, A. and Nørsett, S. P. (2006). From high oscillation to rapid approximation II: Expansions in polyharmonic eigenfunctions, Technical Report 2006/NA07, University of Cambridge.

[13] Iserles, A. and Nørsett, S. P. (2007). From high oscillation to rapid approximation III:Multivariate expansions, Technical Report 2007/NA01, University of Cambridge.

[14] Olver, S. (2007). On the convergence rate of a modified Fourier series, Technical Report 2007/NA02, University of Cambridge.

[15] Poschel, J. and Trubowitz, E. (1987). Inverse Spectral Theory (Academic Press, Boston). 